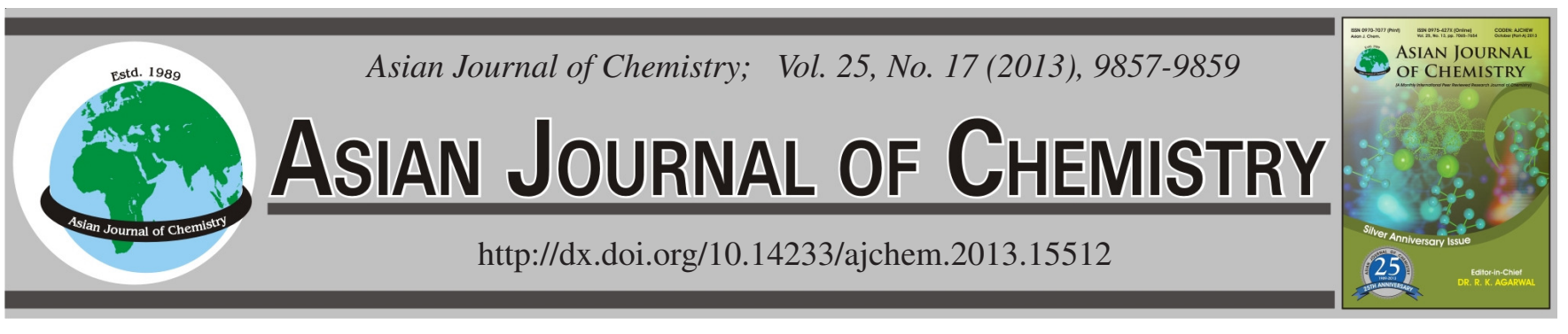

\title{
Ligand-Enhanced Electron Transfer from Zinc Tetraphenylporphyrin to Pyridine-Based Anthraquinone
}

\author{
Li-Zeng Liu ${ }^{1, *}$, XiAn-FAng Meng $^{1}$, Dong-Zhi Liu ${ }^{2}$ and Xue-Qin Zhou ${ }^{2}$
}

${ }^{1}$ College of Science, Tianjin University of Commerce, Tianjin 300134, P.R. China

${ }^{2}$ School of Chemical Engineering, Tianjin University, Tianjin 300072, P.R. China

*Corresponding author: Tel/Fax: +86 22 83692436; E-mail: 1lzeng@ @jcu.edu.cn

(Received: 20 March 2013;

Accepted: 25 October 2013)

AJC-14290

\begin{abstract}
Enhancements of axial ligands on the electron transfer from excited zinc tetraphenylporphyrin (ZnTPP) to pyridine-based anthraquinone (AQPy) were studied in $\mathrm{CH}_{2} \mathrm{Cl}_{2}$ solution at room temperature by UV-visible, fluorescence and fluorescence decay methods. The change in fluorescence lifetime and fluorescence intensity of ZnTPP was found to depend on the formation of the axial ligation with pyridinebased electron acceptor, from 1.70 to $0.16 \mathrm{~ns}$ and $18 \%$, respectively. The intramolecular electron transfer rate constants ( $\kappa_{\mathrm{c}}$ ) values between ZnTPP-AEAQPy were obtained by calculating $\left(\kappa_{\mathrm{c}}=5.7 \times 10^{9} \mathrm{~s}^{-1}\right)$. Pyridine/phene-based anthraquinone derivatives were synthesized and characterized by ${ }^{1} \mathrm{H}$ NMR, IR and ESI-MS.
\end{abstract}

Key Words: Zinc porphyrin, Anthraquinone, Electron transfer, Dyads, Coordination.

\section{INTRODUCTION}

Porphyrins and quinones play a key role as primary electron donors and acceptors in natural photosynthesis ${ }^{1,2}$. There have been numerous investigations of photoinduced electron transfer in artificial porphyrin-9,10-anthraquinone dyads mimicking the function of the P680 primary donor and the $\mathrm{Q}_{\mathrm{A}}$ primary acceptor in biological systems, but the influence of coordination-bond on the electron transfer between electron donors and acceptors has received comparatively little attention in such studies ${ }^{3-7}$. It is important to note that the zinc porphyrin is capable of binding a variety of ligands ${ }^{8}$. Since the binding of the donor-acceptor complex is reversible in nature, after the occurrence of electron transfer, the individual charge-separated species can diffuse away from each other, creating a longlived solvent separated ion pairs in a sufficiently polar medium. Thus, increasing the lifetime of the charge-separated state. In this paper, we wish to report a new coordination-based donoracceptor system, ZnTPP-AQPy, in which the AQPy serves as an electron acceptor and the zinc porphyrin moiety as an electron donor. Compared with the photophysical properties of the mixture of zinc porphyrin and phene-based anthraquinone, the present work provides the enhancements of coordination-bonding on electron transfer from distant zinc porphyrin to pyridine-based anthraquinone.

\section{EXPERIMENTAL}

Zinc tetraphenylporphyrin (ZnTPP) was prepared according to the reported methods ${ }^{9}$. Aminoethyl amino-anthraquinone

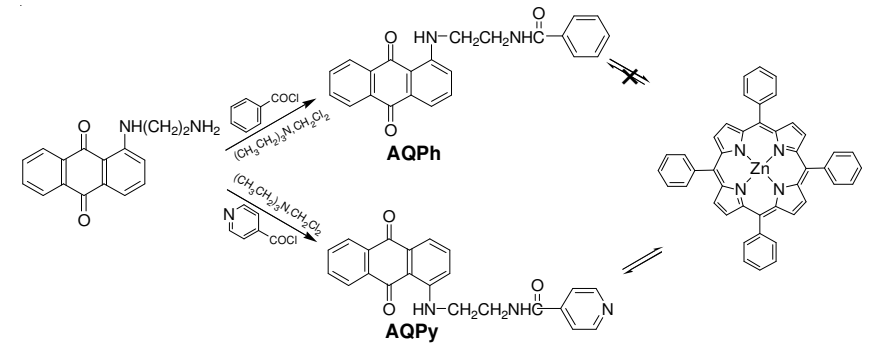

Fig. 1. Synthesis of compound AQPh, AQPy and the equilibrium AQPyZnTPP

(AQ) was synthesized according the method of Wang XQ et al. ${ }^{10}$. All other reagents were analytical grade and further purified by the standard methods if necessary. ${ }^{1} \mathrm{H}$ NMR spectra were recorded on a VARIAN UNITY INOVA 500 spectrometer. ESI mass spectra were obtained with a LCQ Advantage MAX mass spectrometer. Infrared spectra were obtained using a Bio-rad FTS3000. The absorption spectra were performed on a Thermo Spectronic, Helios Gamma UV/visible spectrometer. Steady-state fluorescence spectra were obtained using a Varian CARY ECLIPSE fluorospectrophotometer. Fluorescence lifetime measurements were carried out by time-correlated singlephoton-counting (HORIBA Jobin Yvon IBH spectrometer) at $25^{\circ} \mathrm{C}$.

Preparation of AQPh: In a $50 \mathrm{~mL}$ round-bottom flask, $232 \mathrm{mg}$ (0.6 mmol) AQ, $0.14 \mathrm{~mL}$ (1.2 mmol) benzoyl chloride and $0.4 \mathrm{~mL}(2.9 \mathrm{mmol})$ triethylamine were added to $10 \mathrm{~mL}$ $\mathrm{CH}_{2} \mathrm{Cl}_{2}$ and the reaction mixture was stirred for $2 \mathrm{~h}$ at room temperature. The solvent was removed by rotary evaporation 
and the crude product was dissolved in $5 \mathrm{~mL} \mathrm{CH}_{2} \mathrm{Cl}_{2}$ and was purified by column chromatography on silica gel with $\mathrm{CH}_{2} \mathrm{Cl}_{2}$ : methanol $=5: 1$ as eluent. yield $83 \%$ (Fig. 1). ${ }^{1} \mathrm{H} \mathrm{NMR}\left(\mathrm{CDCl}_{3}\right)$ $\delta: 3.67-3.68\left(\mathrm{~d}, 2 \mathrm{H}, J=2.5 \mathrm{~Hz}, \mathrm{NCH}_{2}\right), 3.80-3.81(\mathrm{~d}, 2 \mathrm{H}, 2.5 \mathrm{~Hz}$, $\left.\mathrm{NCH}_{2}\right), 6.61\left(\mathrm{~s}, 1 \mathrm{H}, \mathrm{CH}_{2} \mathrm{NH}\right), 7.23-7.24(2 \mathrm{H}, J=2.5 \mathrm{~Hz}, \mathrm{ArH})$, 7.42-7.45(m, 2H, ArH) 7.53-7.61(m, 3H, ArH), 7.69-7.81 (m, $3 \mathrm{H}, \mathrm{ArH}), 8.23-8.26$ (t, 2H, ArH), 9.92(s, 1H, -CONH). IR $\left(\mathrm{KBr}, \Delta / \mathrm{cm}^{-1}\right)$ : 3372( -NHCO-), 1655, 1627, $1592(-\mathrm{C}=\mathrm{O})$, 1537, 1510 (-C=C-), 736, 707 (s-Ph). ESI-MS (m/z): 371.1 $[\mathrm{M}+\mathrm{H}]^{+}$.

Preparation of AQPy: In a $50 \mathrm{~mL}$ round-bottom flask, $222 \mathrm{mg}(1.8 \mathrm{mmol})$ isonicotinic acid, $5 \mathrm{~mL}$ thionyl chloride in $15 \mathrm{~mL}$ chloroform was refluxed for $6 \mathrm{~h}$. The cooled reaction mixture was concentrated by rotary evaporation. Then the mixture of $232 \mathrm{mg}(0.6 \mathrm{mmol})$ AQ $0.4 \mathrm{~mL}$ ( $2.9 \mathrm{mmol})$ triethylamine and $10 \mathrm{~mL} \mathrm{CH} \mathrm{Cl}_{2}$ cooled to $0{ }^{\circ} \mathrm{C}$ was slowly added into the intermediate mixture. The reaction mixture was stirred for $2 \mathrm{~h}$ at room temperature. The solvent was removed by rotary evaporation and the crude product was dissolved in $5 \mathrm{~mL}$ $\mathrm{CH}_{2} \mathrm{Cl}_{2}$ and was purified by column chromatography on silica gel with $\mathrm{CH}_{2} \mathrm{Cl}_{2}$ : methanol = 3:1 as eluent. yield $84 \%$ (Fig. 1). ${ }^{1} \mathrm{H}$ NMR $\left(\mathrm{CDCl}_{3}\right) \delta: 3.68-3.69$ (d, $\left.2 \mathrm{H}, J=2.5 \mathrm{~Hz}, \mathrm{NCH}_{2}\right), 3.80-$ $3.81\left(\mathrm{~d}, 2 \mathrm{H}, J=2.5 \mathrm{~Hz}, \mathrm{NCH}_{2}\right), 5.31\left(\mathrm{~s}, 1 \mathrm{H}, \mathrm{CH}_{2} \mathrm{NH}\right), 7.19$ $7.21(\mathrm{~d}, 2 \mathrm{H}, J=2.5 \mathrm{~Hz}, \mathrm{ArH}), 7.55-7.78$ (m, 3H, ArH), 7.91(s, $2 \mathrm{H}, \mathrm{ArH}), 8.23-8.24$ (d, 2H, $J=2.5 \mathrm{~Hz}, \mathrm{ArH}), 8.77$ (s, 2H, ArH), 9.91(s, $1 \mathrm{H},-\mathrm{CONH})$. IR (KBr, $\left.\Delta / \mathrm{cm}^{-1}\right)$ : 3294 (-NHCO-), 1728, 1667, 1638 (-C=O), 1594, 1546, 1505 (-C=C-), 737, 707 $(\mathrm{s}-\mathrm{Ph}) . \mathrm{ESI}-\mathrm{MS}(\mathrm{m} / \mathrm{z}): 371.9[\mathrm{M}+\mathrm{H}]^{+}$.

\section{RESULTS AND DISCUSSION}

Absorption spectra measurements: The absorption spectra of ZnTPP-AQPy are shown in Fig. 2. The evidence of axial coordination to the central zinc in the porphyrin macrocycle was obtained by UV-visible spectra. In the visible spectral region, the absorption spectrum of ZnTPP located at $419 \mathrm{~nm}$ (soret band), 548 and $586 \mathrm{~nm}$ (Q-bands) ${ }^{11}$. Upon incremental addition of an equimolar mixture of AQPy and ZnTPP, the peaks showed a red shift which indicates the axial coordination of the pyridine moiety with $\mathrm{ZnTPP}$ in $\mathrm{CH}_{2} \mathrm{Cl}_{2}{ }^{12}$.

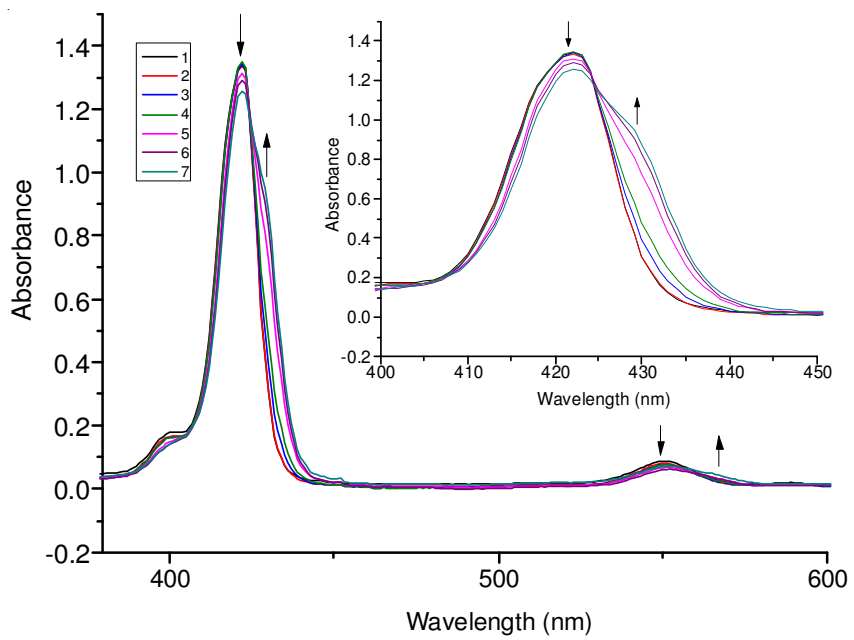

Fig. 2. Absorption spectra of ZnTPP-AQPy in $\mathrm{CH}_{2} \mathrm{Cl}_{2}$, $[\mathrm{ZnTPP}]=4 \times 10^{-6}$ $\mathrm{M},[\mathrm{AQPy}]=0 \mathrm{M}(1), 4 \times 10^{-6} \mathrm{M}(2), 4 \times 10^{-5} \mathrm{M}(3), 8 \times 10^{-5} \mathrm{M}(4)$, $2.4 \times 10^{-4} \mathrm{M}(5), 3.2 \times 10^{-4} \mathrm{M}(6), 4 \times 10^{-4} \mathrm{M}(7)$
Measurment of fluorescence emission spectra: The photoinduced electron transfer from ZnTPP to anthraquinone moiety was studied using the fluorescence emission spectra. The values of relative fluorescence intensities were obtained by the integration of the emission spectra of ZnTPP-AQPh (the mixture of zinc porphyrin and phene-based anthraquinone) and ZnTPPAQPy (the equiluim of zinc porphyrin and pyridine-based anthraquinone) relative to ZnTPP (Fig. 3). When excited at $440 \mathrm{~nm}$, the shape of the fluorescence spectra of ZnTPP-AQPy and ZnTPP-AQPh are the same as that of ZnTPP. However, the fluorescence intensities of axial ligands system are lower than that of ZnTPP. The quenching is highest (18\%) for ZnTPP-AQPy, but the quenching is not observed both in the mixture of tetraphenylporphyrin $(5,10,15,20$-tetraphenylporphyrin)-AQPh and TPP-AQPy (Fig. 4). These results indicate that the photoexcited singlet state of zinc porphyrin is quenched by the axial bonded due to intramolecular electron transfer and no electronic interaction occurs between the porphyrin and the phene based anthraquinone or the tetraphenylporphyrin and the anthraquinone under the photoexcited conditions ${ }^{13}$.

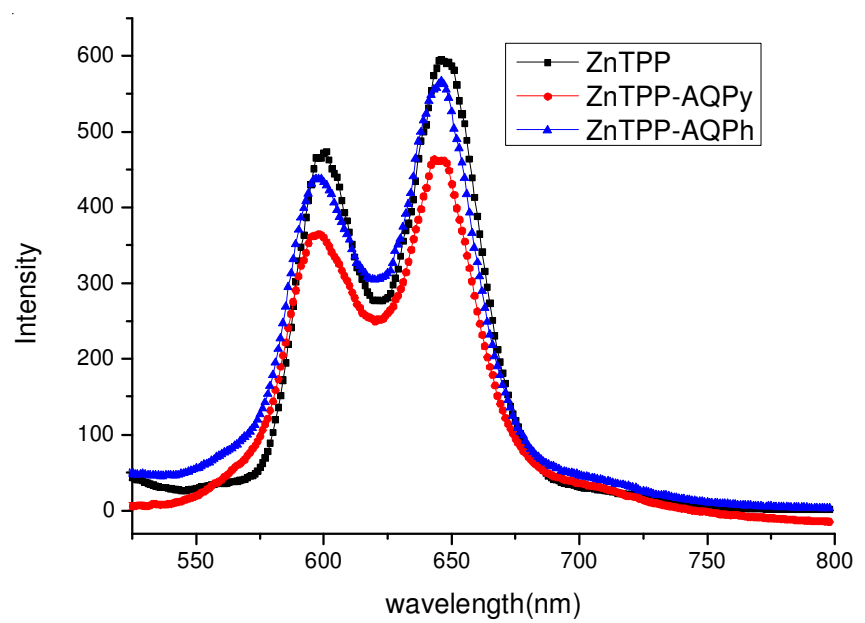

Fig. 3. Fluorescence spectra of ZnTPP, ZnTPP-AQPy and reference sample $\mathrm{ZnTPP}-\mathrm{AQPh}$ in $\mathrm{CH}_{2} \mathrm{Cl}_{2}, 4 \times 10^{-6} \mathrm{M}$

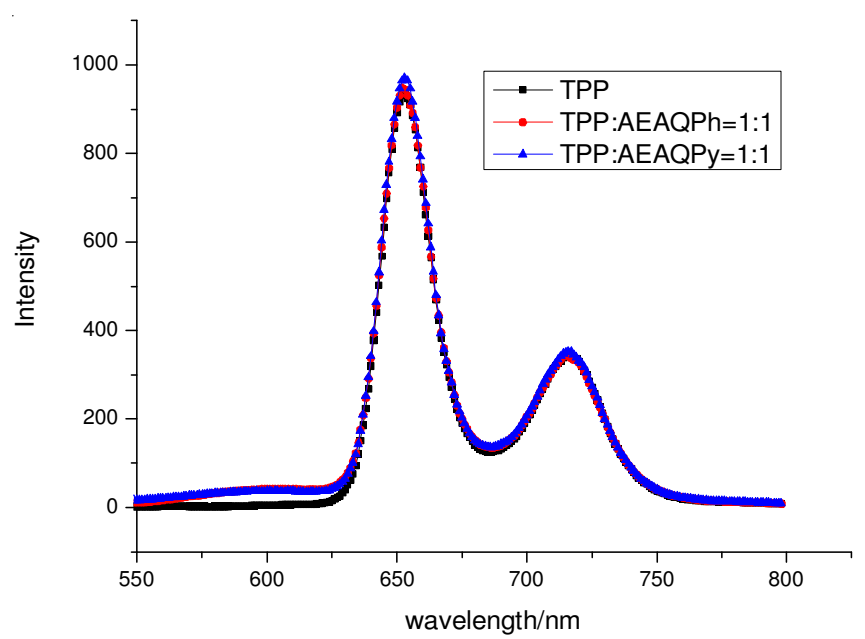

Fig. 4. Fluorescence spectra of TPP, TPP-AQPh and TPP-AQPy (the mixture of TPP and AQPh, AQPy) in $\mathrm{CH}_{2} \mathrm{Cl}_{2}, 4 \times 10^{-6} \mathrm{M}$

Fluorescence lifetime measurements: In Fig. 5 we showed the experimental fluorescence decay curves of ZnTPP, 


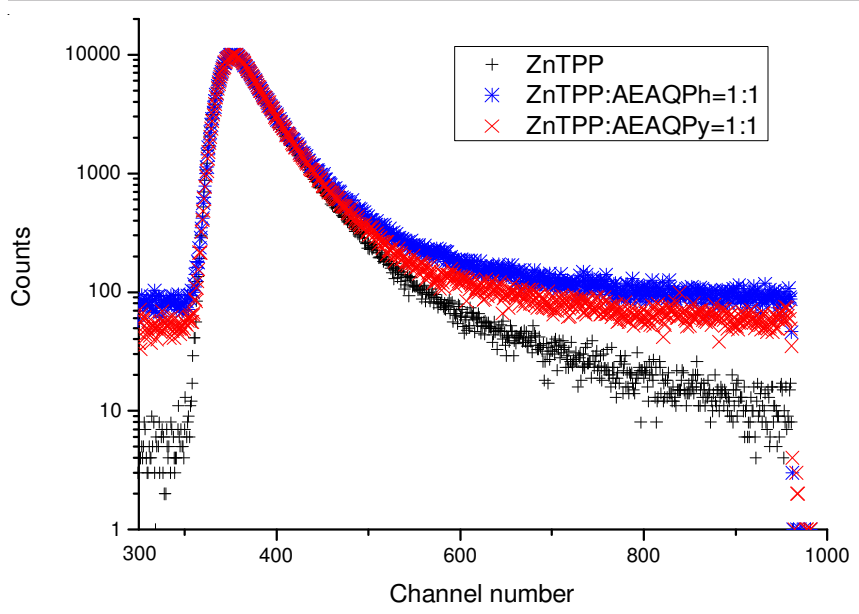

Fig. 5. Fluorescence decay, as measured by nanosecond time correlated single photon counting, for ZnTPP, ZnTPP:AEAQPh = 1:1 and ZnTPP:AEAQPy $=1: 1$ in $\mathrm{CH}_{2} \mathrm{Cl}_{2}$. The concentrations were maintained at $4 \times 10^{-6} \mathrm{M}$ for ZnTPP, $4 \times 10^{-6} \mathrm{M}: 4 \times 10^{-6} \mathrm{M}$ for ZnTPP:AEAQPh = 1:1 and ZnTPP: AEAQPy $=1: 1$. Excitation by $370 \mathrm{~nm}$ nanoled

ZnTPP:AEAQPh = 1:1 and ZnTPP:AEAQPh = 1:1 in $\mathrm{CH}_{2} \mathrm{Cl}_{2}$. The ZnTPP can be fitted perfectly by biexponential fitting with a main component $(90 \%)$ of $1.70 \mathrm{~ns}(\mathrm{CHISQ}=1.05)$. The fit for the mixture of ZnTPP and AEAQPh was fitted by biexponential fitting with a main component $(85 \%)$ of 1.69 ns $($ CHISQ $=1.03)$ and the ZnTPP-AEAQPy was also fitted perfectly by biexponential fitting with a main component $(93 \%)$ of $0.16 \mathrm{~ns}(\mathrm{CHISQ}=1.03)$. The lifetime became shorter for zinc porphyrin with AEAQPy. Therefore, the fluorescence decay may arise from the direct quenching of the photoexcited singlet state of the porphyrin unit to the anthraquinone moiety ${ }^{14,15}$. From the lifetimes obtained from the fluorescence decay profiles, intramolecular electron transfer rate constants $\left(\kappa_{\mathrm{c}}\right)$ were calculated by the following equation ${ }^{14}: \kappa_{\mathrm{c}}=1 / \psi-1 / \psi_{0}$, where $\psi_{0}$ is the fluorescence lifetime of the ZnTPP. From a comparison of $\kappa_{\mathrm{c}}$ values between ZnTPP: AEAQPy $\left(\kappa_{\mathrm{c}}=5.7\right.$ $\left.\times 10^{9} \mathrm{~s}^{-1}\right)$ and ZnTPP: AEAQPh $\left(\kappa_{\mathrm{c}} \approx 0\right)$, the $\kappa_{\mathrm{c}}$ value of pyridine compound is higher than that of phene compound. In pyridine compound the AEAQPy can come closer to the porphyrin ring by coordination bond compared with phene compound and the electron may transfer more easily.

\section{Conclusion}

As was to be expected from thermodynamic considerations ZnTPP-AQPy exhibits photoinduced electron transfer reactivity, in which the ternary complex can hold tightly a donor (ZnTPP) and an acceptor (AQPy) together via an axial coordination between the two moieties. In dichloromethane fluorescence quenching of ZnTPP by pyridine-based anthraquinone occurs in the ternary complex than intermolecular processes between the uncomplexed molecules(ZnTPP-AQPh , TPP-AQPy and TPP-AQPh). Enhanced electron transfer has been observed in the supramolecular ternary complex by the decreasing fluorescence lifetime of ZnTPP. The method of constructing multicomponent assemblies facilitating electron transfer among the components via a coordination bond provides a powerful way to construct systems with increasing complexity involving many components, keeping their structures and functions under control.

\section{ACKNOWLEDGEMENTS}

The authors acknowledged the support of the National Natural Science Foundation of China (No. 31271935) and the Tianjin University of Commerce (No. 090114).

\section{REFERENCES}

1. I. Bertini, H.B. Gray, E.I. Stiefel and J.S. Valentine, Biological Inorganic Chemistry, University Science Books: Sausalito, CA (2007).

2. G. Renger and T. Renger, Photosynth. Res., 98, 53 (2008).

3. M.R. Wasielewski, Chem. Rev., 92, 435 (1992).

4. D. Wróbel, J. Lukasiewicz and H. Manikowski, Dyes Pigm., 58, 7 (2003).

5. M.L. Tao, X.Q. Zhou, J. Ma, D.Z. Liu and J. Xing, Dyes Pigm., 75, 408 (2007).

6. M.L. Tao, L.Z. Liu, D.Z. Liu and X.Q. Zhou, Dyes Pigm., 85, 21 (2010).

7. V. Balzani, Electron Transfer in Chemistry, VCH Wiley: Weinheim, vol. 3 (2001).

8. M.E. El-Khouly, O. Ito, P.M. Smith and F. D'Souza, J. Photochem. Photobiol. C: Rev., 5, 79 (2004).

9. A.D. Adler, F.R. Longo, F. Kampas and J. Kim, J. Inorg. Nucl. Chem., 32, 2443 (1970).

10. X.Q. Wang, Chem. J. Chin. Univ., 18, 834 (1997).

11. P.C. Dave and D. Srinivas, J. Porphyrins Phthalocyan., 2, 243 (1998).

12. J. Otsuki, M. Takatsuki, M. Kaneko, H. Miwa, T. Takido, M. Seno, K. Okamoto, H. Imahori, M. Fujitsuka, Y. Araki, O. Ito and S. Fukuzumi, J. Phys. Chem. A, 107, 379 (2003).

13. S.K. Sugunan, B. Robotham, R.P. Sloan, J. Szmytkowski, K.P. Ghiggino, M.F. Paige and R.P. Steer, J. Phys. Chem. A, 115, 12217 (2011).

14. I. Okura and H. Hosono, J. Phys. Chem., 96, 4466 (1992).

15. D. Kowalska and R.P. Steer, J. Photochem. Photobiol. A, 195, 223 (2008). 